\title{
EP-188
}

\section{Preoperative body mass index and amylase level predict postoperative pancreatic fistula independently}

\author{
Deng NING ${ }^{1}$, Li JIANG ${ }^{* 2}$, Xiaoping CHEN' ${ }^{1}$, Chao WANG \\ ${ }^{1}$ Hepatic Surgery Center, Affiliated Tongji Hospital, Tongji Medical College, Huazhong University of Science and Technology, Wuhan, China \\ ${ }^{2}$ Department of Biliary and Pancreatic Surgery, Affiliated Tongji Hospital, Tongji Medical College, \\ Huazhong University of Science and Technology, Wuhan, China
}

Introduction: Postoperative pancreatic fistula (POPF) is one of the difficult complications after distal pancreatectomy (DP), resulting increased postoperative morbidity and mortality. It is still a hotspot to prevent the occurrence of POPF in recent researches. Our study aimed to investigate the risk factors of pancreatic fistula in patients following DP.

Methods: We retrospectively analyzed 206 patients with DP in Tongji Hospital, China, from January 2014 to March 2019. Univariate and multivariate logistic regressions were performed to assess the risk factors of POPF.

Results: A total of 206 eligible patients were enrolled into this study, including 123 females and 83 males. The overall morbidity was $30.1 \%$. Postoperative complications included postoperative biliary fistula (0.5\%), delayed gastric emptying (1\%), grade B pancreatic fistula (4.9\%), grade C pancreatic fistula (1.9\%), postoperative abdominal abscess (2.4\%), wound infection $(3.4 \%)$, percutaneous drainage $(18.4 \%)$ and reoperation $(0.5 \%)$. The first hospitalization time was 24 days. There were 21 patients re-hospitalized within 30 days and 8 patients within 90 days. No postoperative death was observed. Univariate analysis indicated that the occurrence of POPF was related to preoperative BMI $>25 \mathrm{~kg} / \mathrm{m}^{2}(p=0.034)$, serum pancreatic amylase $>53 \mathrm{U} / \mathrm{L}(p=0.049)$, splenic preservation $(p=0.069)$ and splenic vessel preservation $(p=0.036)$. Multivariate analysis showed that preoperative $\mathrm{BMI}>25 \mathrm{~kg} / \mathrm{m}^{2}(\mathrm{OR}=2.637, p<0.05)$ and serum pancreatic amylase $>53 \mathrm{U} / \mathrm{L}(\mathrm{OR}=2.658, p<0.05)$ were independent risk factors of clinical related-postoperative pancreatic fistula.

Conclusions: Preoperative BMI $>25 \mathrm{~kg} / \mathrm{m}^{2}$ and serum amylase $>53 \mathrm{U} / \mathrm{L}$ are independent risk factors of POPF. It is necessary to monitor BMI and amylase level preoperatively in patients undergoing DP. 